\title{
The Effect of Reading Direction on Emotion Recognition: A Pilot Study
}

Vargha B* and Labadi B

Department of Psychology, University of Pecs, Hungary

*Corresponding author: Balint Vargha, Ph.D. student, Department of Psychology, University of Pecs, Hungary, Tel: +36 72501 516; Email: varghabalint@gmail.com

Research Article

Volume 3 Issue 1

Received Date: January 25, 2019

Published Date: June 17, 2019

DOI: $10.23880 / \mathrm{mhrij}-16000126$

\section{Abstract}

In this pilot study we tested whether or not the direction of reading (independently from its content) influences participants' emotional experience. We tested a small sample of 30 Japanese people in a repeated measure setting. Participants completed a short emotion-recognition task following two different reading direction conditions: left-toright and top-to-bottom. Results show that reading direction tends to have an impact on recognition of expression of emotion. Based on these positive results, we believe further research is necessary to verify the observed effects and to refine present findings.

Keywords: Reading Direction; Reading; Perception; Emotion Recognition; Facial Expressions

Classification codes: 2340,2360

Abbreviations: VT: Valence Theory; RVF: Right Visual Field; LVF: Left Visual Field; BPT: Bilateral Projection Theory; SFT: Split Fovea Theory; LH: Left Hemisphere; RH: Right Hemisphere; PVL: Preferred Viewing Location; OVP: Optimal Viewing Point; VWF: Visual Word Form; VH: Valence Hypothesis; RHH: Right Hemisphere Hypothesis; VF: Visual Field; LR: Left-to Right; RL: Right-to Left; TB: Top-to-Bottom; AWS: Approach/Withdrawal System; JAFFE: Japanese Female Facial Expression; ARME: Asian Reading the Mind in the Eyes; RME: Reading the Mind in the Eyes

\section{The Effect of Reading Direction on Emotion Recognition}

Research shows that unilateral physical stimulations can influence emotional experiences. Besides research on the effects of handedness on behavior and personality, studies have used an experimental setting designed to show a connection of this kind using nostril breathing, face and hand muscles, and vibrotactile stimulations. In a series of studies, Schiff and Lamon $(1989,1994)[1,2]$ demonstrated that unilateral stimulations induce different emotional states, depending on the side of the stimulation. A right-sided stimulation induced a positive emotional experience, while a left-sided stimulation induced a negative emotional experience. These findings are in line with the well-based valence theory (VT). Schiff and Lamon argued that unilateral physical stimulation falls under the control of the contralateral hemisphere, which then affects emotional experience according to the side of activation.

In our research we wanted to test whether or not the direction of reading as a physical stimulation has an effect similar to the other forms of unilateral stimulations 


\section{Mental Health \& Human Resilience International Journal}

previously studied by Schiff and Lamon. We believe it is important to test the possibility of this connection because we spend a lot of time reading every day. Therefore, if this effect exists and can be specified, then we need to understand this influence. Further, cultures have different reading habits with different directions of reading. If the direction of reading affects emotional experience, this could produce different interpretations of the emotional content of the same event, leading to misunderstandings between cultures with different reading habits.

Following, first we will discuss how the act of reading is similar to a unilateral physical stimulation, then we move on and discuss the two models of hemispheric lateralization of emotion processing that were used to formulate hypotheses in this experiment. After that we will describe how and why the experiment was conducted and form hypotheses based on our assumptions and the emotion-processing models. After the method and results section, we discuss our findings where we propose a possible explanation for our results, and finally we introduce other possible topics for further research related to reading direction and hemispheric laterality. Note that this is a pilot study so the conclusions drawn from this experiment are very limited and are more like possible perspectives than irrefutable facts, which require further research for validity.

\section{Hemispheric Processing of Words and Sentences}

The role and function of visual fields (especially the foveal region) are important and well-studied topics in vision research of reading. Although it is an accepted fact that parafoveal regions project contralaterally, there is an ongoing debate about whether the foveal region functions as a whole in which information is projected to both hemispheres or whether information is processed laterally similar to parafoveal vision [3-5].

On the sentence level, contralateral projection to the hemispheres is well supported by evidence found by Pollatsek, Bolozky, Well, and Rayner (1981) [6] who arrived to the conclusion that English readers' perceptual span is asymmetric in a way that information falling to the right of the fixated word is more extensive (15-16 characters) than that falling to the left side (3-4 characters), thus occupying more area in the right visual field (RVF) than the left visual field (LVF). (The effect is reversed for readers reading Hebrew text, which is read from right-to-left.)
On the word (foveal) level, however, the picture is not so clear. There are two competing theories about hemispheric projection linked to the foveal region. The bilateral projection theory (BPT) posits that the information from the fovea region will project both to the left and right hemispheres regardless the point of fixation [4]. The split fovea theory (SFT) argues that the fovea is split in the middle, with information to the right of the center of the fovea projecting to the left hemisphere (LH) and information to the left of the center of the fovea projecting to the right hemisphere (RH) [3].

\section{Reading and Hemispheric Processing}

When we read, our fixation typically falls to the initial one-third of the word, called the preferred viewing location (PVL). This also is where fastest and most accurate word recognition occurs and is the optimal viewing point (OVP) for word identification [7-9]. If the BPT is correct, then word-level information processing is done by both hemispheres equally, while sentence-level processing is done mostly by the LH. If the SFT is the valid theory, however, then processing is mostly done by the LH on both the word and sentence level. Whichever the case, the RVF and the LH are used more in reading than the LVF and the RH; therefore, we consider the direction of reading to be possibly an asymmetric physical stimulation in the case of (normal, everyday) left-to-right reading.

\section{RVF Superiority in Reading}

The RVF superiority in reading found in the previous experiments is attributed mainly to the hemispheric specialization of the LH for processing language-related stimuli (i.e., the Broca area and the visual word form [VWF] area), but growing evidence suggests that it is, in part, a result of perceptual learning. In a series of experiments, Nazir, Ben-Boutayab, Decoppet, Deutsch, and Frost (2004) [10] reinforced this supplementary explanation. They argued that because of the time people spend "practicing" discriminating orthographic material and because of the regular use of the close-to-center landing site, reading improves in a way such that people will process more and more information in the visual field identical to the direction of visual scanning (i.e., in left-toright reading the right visual field). Because of that, those who read in a left-to-right direction for a long time will use their RVF more and more compared to their LVF, thus making their LH more active compared to their RH while reading.

Similarly, reinforcing the previous assumption Wong and Hsiao (2012) [11] found that the OVP effect for 


\section{Mental Health \& Human Resilience International Journal}

reading musical notes was a consequence of long-term reading practice of musical notes. Novice, intermediate, and expert musicians read three-note sequences in the experiment. Their fixation point fell more and more to the left of the center of the sequence as their level of experience increased. From these results, it seems likely then that the LH's superiority in processing written material directed in a left-to-right direction is partly due to a learning process, namely to our habit of reading in this direction. In this case the scale of the asymmetry created by left-to-right reading is the product of a learning mechanism instead of a pure, innate brain activation mechanism. Now, we take a small detour to familiarize with emotion processing theories then we move on to make our hypothesis based on our assumptions.

\section{Hemispheric Processing of Emotions}

Hemispheric lateralization of emotions remains a debated theory in the field of cognitive neuroscience despite several studies that used varied methods in an attempt to support cerebral asymmetries. Currently two competing theories attempt to explain these findings: the valence hypothesis (VH) and the right hemisphere hypothesis (RHH).

According to the $\mathrm{VH}$, unilateral stimulations activate the contralateral hemisphere, which then affects emotional experience (e.g., mood, performance). During a left-sided stimulation, the right hemisphere, which is specialized in negative emotion processing, will be activated. During a right-sided stimulation, the left hemisphere, which is specialized in positive emotion processing, will be activated. The RHH, however, suggests that the right hemisphere is responsible for all emotion processing, regardless of valence.

Researchers commonly try to deliberate between these two theories by using visual field (VF) experiments in which activation is attributed to the hemisphere opposite the VF (i.e., if the stimulus is presented in the $\mathrm{RVF}$, then its processing is attributed to the $\mathrm{LH}$ ).

Despite the many research studies conducted in this area, the results are still inconclusive. On one hand, there are findings supporting the theory that positive emotions are better and faster recognized when presented in the RVF (i.e., the left hemisphere processes positive emotions) and vice versa for negative emotions [12-14]. On the other hand, in a divided visual field experiment, when the stimuli of facial expressions are simultaneously presented in both the right and left visual fields, participants tend to recognize the target expression better and faster when it is presented in the LVF [15-17]. The findings of these studies have been interpreted in favor of either the $\mathrm{VH}$ or the RHH, so the debate remains unresolved.

\section{Physical Stimulations and Emotions}

Schiff and Lamon conducted a series of experiments in which emotional processing was not tested by stimuli presentations to visual fields but by using physical stimulations to generate emotional experience. They were among the first researchers who found a laterality effect between physical activity and emotional experience. Small unilateral muscle contractions of the face [1] and the hand [2], unilateral nostril breathing [18], and unilateral vibrotactile stimulations [19] all influenced emotional experience, depending on the side of contraction. Stimulations on the left side resulted in negative states of emotion while stimulations on the right side resulted in positive states of emotion. The authors explained their results on the basis of the $\mathrm{VH}$.

\section{Present Research}

The goal of this research was twofold. First, based on our theoretical background that VF use is unequal during reading, we used the reading process as a physical stimulation to address the inconsistent findings of lateralized emotional processing, and at the same time prove that direction of reading can function as a unilateral stimulation. We expected that the act of reading would generate distinguishable emotional processing reaffirming its unilateral stimulating nature and that it would reaffirm or deny the competing models of hemispheric lateralization of emotions.

Considering the SFT theory and the results of Pollatsek et al. (1981) [6], we hypothesized that the asymmetric use of VFs during reading would generate a lateralized hemispheric activation that could be captured through emotion processing as in the Schiff and Lamon experiments.

Schiff and Lamon (1989, 1994) [1,2] induced stimulations of both the right and left side and then compared the effects. In the current study, however, it was difficult to set up a similar inverse condition (i.e., leftto right [LR] reading vs. right-to left [RL] reading) because the capacity to read in a different direction was lacking. Incapable of constructing an experimental situation where LR and RL reading conditions could be compared on the same sample in a within-subject design, we chose to compare the conditions of LR and top-to-bottom (TB) reading. Japanese can be read in both LR and TB 


\section{Mental Health \& Human Resilience International Journal}

directions, using the same graphemes. Moreover, in this setting, the sample shared the same lingual and cultural background contrary to settings in which two languages are read by the same person [6] or in which different languages are read by different people. Kajii and Osaka (2000) [20] found that the OVP effect disappeared during TB reading in a Japanese sample, which means that while reading in a TB direction both VFs are used equally for information processing, preventing the hemispheric lateralization from occurring.

For testing, we chose a facial expression recognition task because it is easy to measure and the results are simple to interpret. We made the following predictions based on information from competing emotionprocessing models such as the $\mathrm{VH}, \mathrm{RHH}$, and the approach/withdrawal system (AWS) theory, and from studies by Demaree, Everhart, Youngstrom, and Harrison (2005), Fusar-Poli et al. (2009), and Harmon-Jones, Vaughn-Scott, Mohr, Sigelman, and Harmon-Jones (2004) [21-25].

First, due to the hemispheric lateralization created by reading, we expected that the left-weighted asymmetric use of the LH during LR reading would result either in superior and faster recognition of emotions that are attributed to the LH (e.g., anger ${ }^{1}$, happiness, surprise) or inferior emotion recognition in general in concordance with the RHH.

Second, in the TB reading condition where the visual fields are used evenly for information processing, we expected either better performance and faster recognition of emotions attributed to the RH (e.g., sadness, disgust, fear) or superior emotion recognition in general in accordance with the RHH.

\section{Method}

\section{Participants}

Thirty Japanese people (17 men and 13 women) participated in the experiment. Their mean age was 30.8 $(S D \pm 6.228)$. They were all residents of Tokyo and most of them were studying at Hitotsubashi University. Recruitment was done both through advertisement and

\footnotetext{
${ }^{1}$ We attributed anger to the LH based on studies conducted by HarmonJones and Allen (1998), Harmon-Jones, Sigelman, Bohling, and Harmon-Jones (2003), and Harmon-Jones et al. (2004).
}

personal inquiry. Those who signed up for the experiment via advertisement received $1500 ¥$ per session.

\section{Materials}

The stimulus material consisted of two standardized test batteries that measure basic emotion recognition. The first was the Japanese Female Facial Expression (JAFFE) measure by Lyons, Akamatsu, Kamachi, and Gyoba (1998) [26]. The second was the Asian Reading the Mind in the Eyes (ARME) instrument by Adams et al. (2010) [27], which was used to measure more complex emotion and state recognition.

Image material (Stimuli): We selected 48 pictures from the JAFFE database: six faces for each basic emotion and 12 neutral faces. The JAFFE images are of female actors showing facial expressions of the six basic emotions and neutral expressions.

We administered all 36 pictures from the ARME, which is an adaptation of the Reading the Mind in the Eyes (RME) test originally developed by Baron-Cohen, Wheelwright, Hill, Raste, and Plumb (2001) [28] to measure mind reading capabilities (considered an advanced theory of mind test of typically developed adults and adults with an autism spectrum disorder. We decided to use ARME pictures as a general emotion recognition variable because they present complex emotional and mental states [29] and their valence is less confined than basic emotions, thus allowing a less valence-specific processing. Participants were presented a series of images of the eye region of the face of an equal number of female and male actors. Four complex mental state descriptors were presented around each image, one at each corner, with one being the correct target word describing the mental state of the actor. Participants were instructed to choose the word that best described what the person in the photo was thinking or feeling.

The images for both batteries were presented on a 13inch laptop screen, administered by either DMDX (for Windows) or Psyscope (for Mac) software. The instructions and the emotion labels used during the presentation were written in the same direction as the text the participants read during the reading task.

The pictures were grouped into two image sets in order to be able to measure the performance of the same test participant on both reading conditions. Each set contained three JAFFE pictures of each of the six basic emotions, six JAFFE pictures of neutral faces, and 18 ARME pictures. 


\section{Mental Health \& Human Resilience International Journal}

The tests were scored by totaling the number of items (images) for which the participant correctly identified the mental state and basic emotion portrayed. Reaction times also were registered for each image task.

Reading material (conditions): In the experiment, we used four reading texts that differed in two dimensions: their content (Text A or Text B) and their direction of reading (left-to-right or top-to-bottom). This created the following conditions: (a) Text A read from left to right, (b) Text A read from top to bottom, (c) Text B read from left to right, and (d) Text B read from top to bottom. The texts were 24-25 pages long and were put together from randomly selected tales from a Japanese website. All tales appeared in only one of the texts and were written in Japanese Hiragana.

\section{Procedure}

Each participant was tested individually in a soundproof room during two sessions. During the first session, participants listened to Sergei Rachmaninoff's Allegro Vivace for 3 minutes to ensure a similar mood for each person before starting the experiment. Then the participants were given one of the compilations of Japanese tales to read for 30 minutes. In the second session, participants completed the facial expression recognition task involving 24 pictures from the JAFFE database and 18 pictures from the ARME test. The experiment was repeated a week later. The order of the conditions (including image sets, starting condition, and reading materials) was randomized and counterbalanced.

\section{Statistical Method}

Response times from trials with errors were excluded, and those response times greater than two standard deviations above the mean were ignored as outliers. We ran a repeated measures ANOVA for both performance and response time data of the ARME and JAFFE pictures. For the ARME pictures, we used a mixed 2x2 design, in which the within-subject variable was reading direction and the between-subject variable was gender. For the
JAFFE pictures, we added emotion as a third dimension, resulting in a $2 \times 2 \times 7$ design. We also ran separate repeated measures ANOVA for each emotion, using the same mixed $2 \times 2$ design with reading direction as the within-subject variable and gender as the betweensubject variable.

\section{Results}

The analyses yielded several significant results for reading direction as shown in Table 1 . Besides the main effect between the two reading conditions in performance for emotion recognition for the JAFFE pictures $(F[1,28]=$ 4.884), there were significant differences in the recognition of angry $(F[1,28]=18.545)$, sad $(F[1,28]=$ $26.693)$, and neutral faces $(F[1,28]=9.967)$. There also was a tendency for difference in recognition of fearful faces $(F[1,28]=3.332)$.

Although LR readers performed better on recognizing basic emotions in general and three basic emotions in specific (anger, sadness, and fear), TB readers were superior at recognizing faces showing a neutral expression (Figure 1). In addition, LR readers tended to be faster in general in their judgment for ARME pictures $\left(F[1,28]=3.624, p=.067, \mu^{2}=.115\right.$, and $M D=-959[-$ $1991,73])$ while TB readers showed a tendency to be faster specifically for fearful faces $\left(F[1,28]=3.89, \mu^{2}\right.$ $=.122, p=.059$, and $M D=392[-15,800]$ ) (Figure 2).

The analysis showed only one difference related to gender. Women showed a tendency to recognize fearful faces faster than men $\left(F[1,28]=3.155, \mu^{2}=.106, p=.087\right.$, and $M D=.339[-.042, .721])$.

Interestingly, we found an unexpected interaction between gender and reading direction in the performance scores for happy faces $\left(F[1,28]=6.283, \mu^{2}=.183, p<.05\right)$ (see Figure 3). The interaction between the two variables is significant. Although men are better at recognizing happy faces in the LR reading condition, the same is true for women in the TB reading condition.

\begin{tabular}{|c|c|c|c|c|c|c|c|c|}
\hline & \multicolumn{2}{|c|}{ MS for LR group } & \multicolumn{2}{|c|}{ MS for TB group } & \multicolumn{2}{|c|}{ ES } & \multicolumn{2}{|c|}{ MD and $95 \% \mathrm{CI}$} \\
\hline & Perf & RT & Perf & RT & Perf & RT & Perf & RT \\
\hline \multirow{2}{*}{ ARME } & 12.73 & 6871 & 12.97 & 7826 & \multirow{2}{*}{$\mu^{2}=.007$} & \multirow{2}{*}{$\mu^{2}=.115^{t}$} & -0.206 & -959 \\
\hline & $(2.42)$ & (2079) & $(2.16)$ & (3377) & & & {$[-1.125, .713]$} & {$[-1991,73]$} \\
\hline \multirow{2}{*}{ JAFFE } & 2.13 & 3976 & 2 & 4049 & \multirow{2}{*}{$\mu^{2}=.149^{*}$} & \multirow{2}{*}{$\mu^{2}=.008$} & 0.127 & -72 \\
\hline & $(0.41)$ & (123) & $(0.51)$ & (171) & & & {$[.009, .245]$} & {$[-389,245]$} \\
\hline \multirow{2}{*}{ Happiness } & 2.13 & 3299 & 1.97 & 3632 & \multirow{2}{*}{$\mu^{2}=.015$} & \multirow{2}{*}{$\mu^{2}=.068$} & 0.111 & -328 \\
\hline & $(0.78)$ & (731) & $(0.62)$ & (1205) & & & {$[-.231, .453]$} & {$[-799,143]$} \\
\hline Surprise & 2.47 & 4341 & 2.6 & 4182 & $\mu^{2}=.031$ & $\mu^{2}=.008$ & -0.145 & -146 \\
\hline
\end{tabular}

Vargha B and Labadi B. The Effect of Reading Direction on Emotion Recognition: 
Mental Health \& Human Resilience International Journal

\begin{tabular}{|c|c|c|c|c|c|c|c|c|}
\hline & $(0.78)$ & (1177) & $(0.5)$ & (1189) & & & {$[-.458, .168]$} & {$[-787,494]$} \\
\hline \multirow{2}{*}{ Sadness } & 2.1 & 3562 & 1.5 & 3721 & \multirow{2}{*}{$\mu^{2}=.448^{* * *}$} & \multirow{2}{*}{$\mu^{2}=.009$} & 0.602 & 192 \\
\hline & (1) & (1148) & $(0.78)$ & (1647) & & & {$[.363, .840]$} & {$[-605,988]$} \\
\hline \multirow{2}{*}{ Anger } & 2.47 & 3746 & 1.77 & 4190 & \multirow{2}{*}{$\mu^{2}=.400^{* * *}$} & \multirow{2}{*}{$\mu^{2}=.049$} & 0.708 & -473 \\
\hline & $(0.57)$ & (1175) & $(1.17)$ & (2111) & & & {$[.372,1.044]$} & {$[-1283,336]$} \\
\hline \multirow{2}{*}{ Fear } & 1.7 & 4951 & 1.37 & 4561 & \multirow{2}{*}{$\mu^{2}=.106^{t}$} & \multirow{2}{*}{$\mu^{2}=.122^{t}$} & 0.339 & 392 \\
\hline & (1.21) & $(826)$ & $(0.93)$ & (742) & & & {$[-.042, .721]$} & {$[-15,800]$} \\
\hline \multirow{2}{*}{ Disgust } & 1.77 & 4416 & 1.37 & 4359 & \multirow{2}{*}{$\mu^{2}=.087$} & \multirow{2}{*}{$\mu^{2}=.004$} & -0.387 & 91 \\
\hline & $(0.86)$ & (1077) & $(0.68)$ & (1385) & & & {$[-.872, .098]$} & {$[-486,668]$} \\
\hline \multirow{2}{*}{ Neutral } & 4.68 & 3608 & 5.36 & 3837 & \multirow{2}{*}{$\mu^{2}=.263^{* *}$} & \multirow{2}{*}{$\mu^{2}=.023$} & -0.338 & -233 \\
\hline & $(0.23)$ & (1754) & $(0.16)$ & (1527) & & & {$[-.558,-.119]$} & {$[-826,360]$} \\
\hline
\end{tabular}

Note. Significant differences are marked with asterisks and tendencies with a t.

${ }^{\mathrm{t}} p<.1,{ }^{*} p<.05,{ }^{* *} p<.01,{ }^{* * *} p<.001$

Table 1: Performance and Response Time (RT) Mean Scores.

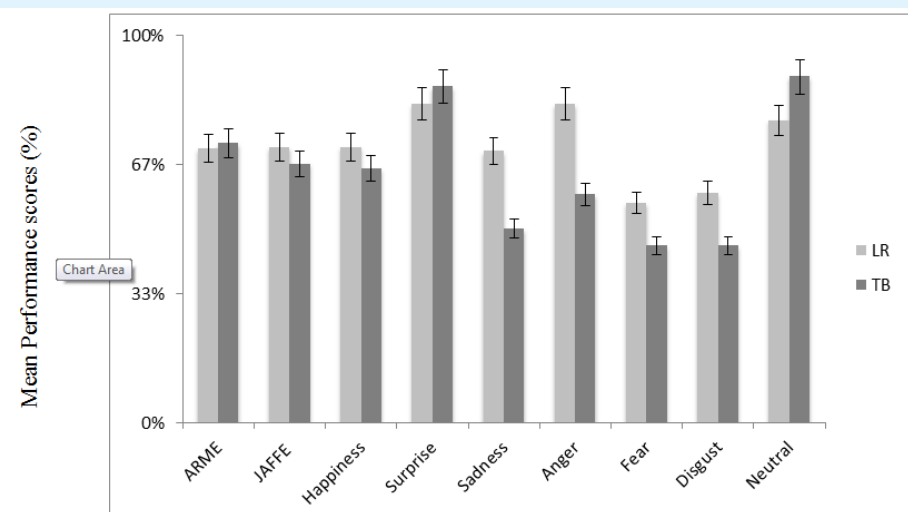

Note: Error bars indicate a 95\% confidence interval.

Figure 1: Mean performance scores for ARME and JAFFE pictures in two reading conditions.

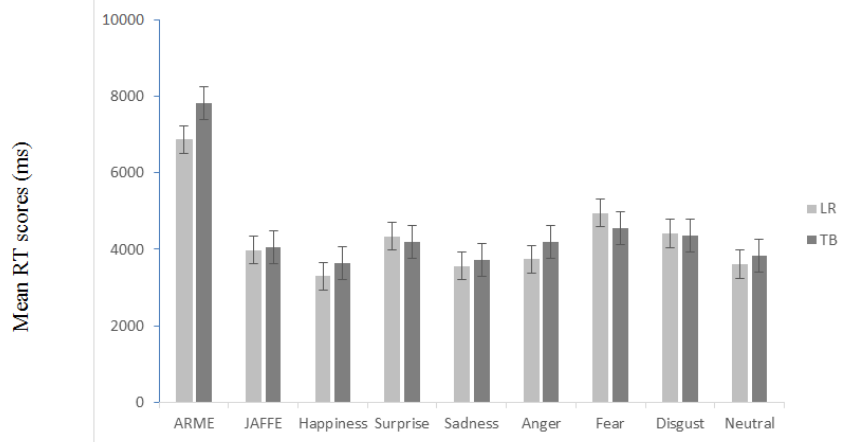

Note: Error bars indicate a 95\% confidence interval.

Figure 2: Mean response time scores for ARME and JAFFE pictures in two reading conditions. 


\section{Mental Health \& Human Resilience International Journal}

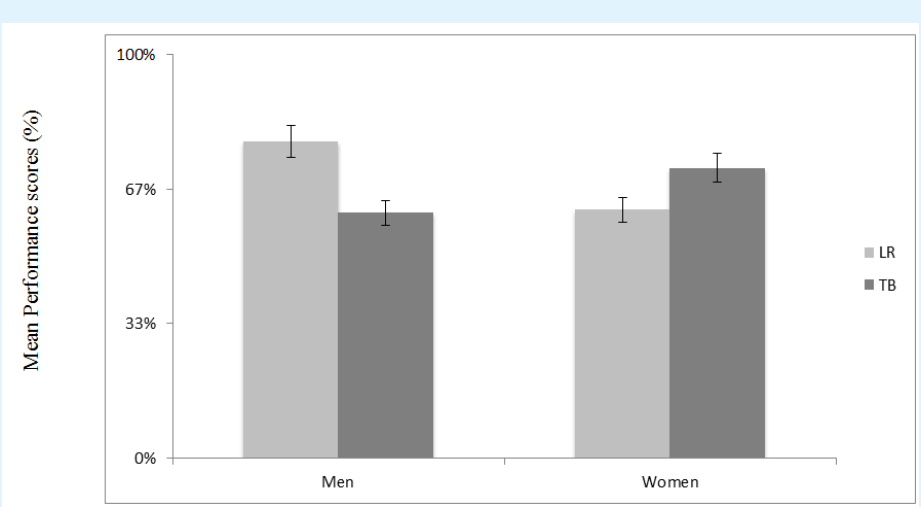

Note: Error bars indicate a 95\% confidence interval.

Figure 3: Mean performance scores for pictures showing happy facial expressions for men and women in both reading conditions.

\section{Discussion}

First, before discussing our results it is important to remind the reader that this experiment was a pilot study with only a small sample size and a low number of trials so the conclusions drawn from this experiment are very limited and needs further research for its verification and refinement.

Keeping the previous in mind, from our results it seems that reading direction influence emotion recognition. In the case of three basic emotions (anger, sadness, and fear), the LR reading condition resulted in better recognition than the TB reading condition. This also was true when we compared all the correct responses for all the JAFFE pictures. Conversely, participants using the TB reading condition were better at discerning neutrality.

Our results do not fit with our initial hypotheses based on current emotion-processing theories and results. The LR reading condition did not result in quicker judgments of approach-relevant emotions such as anger and happiness, and better performance among participants was not restricted to these emotions either. In our experiment, participants in the LR reading condition performed better at recognizing anger, sadness, and fear, and they were faster at recognizing the ARME pictures, which measured general emotion perception.

Similarly, contrary to our prediction, participants in the TB reading condition were not faster nor better at recognizing both of the two withdrawal-related emotions (fear and disgust), but were only faster when recognizing fear. In addition, they were not better at identifying any emotion, including ARME pictures. Instead, they were unexpectedly better at recognizing neutral faces (i.e., those free of valence).

Despite the inconsistency between our hypothesis and results we can propose a possible explanation. According to Killgore and Yurgelun-Todd's (2007) [30] working model, the right hemisphere is responsible in early emotion processing regardless of valence, while the left hemisphere activates valence-specific functions in the brain. In light of this theory, higher performance scores found in the LR reading condition for valence-specific pictures could be explained by the assumed higher LH activation (compared to the RH). Likewise, the higher performance scores for neutral faces found for the TB reading condition could be due to the more symmetric activation of the hemispheres. It remains unknown from this pilot study however, why anger, sadness, and fear were recognized more accurately than surprise, happiness, and disgust.

In addition, Killgore and Yurgelun-Todd's model can account for the slower response time during the recognition of ARME pictures in the TB reading condition. It is possible that although LR reading helps in attaching valence to pictures through a higher LH activation, thus accelerating the recognition process, TB reading slows this down by rendering the facial expression on the picture back to neutral valence through a more symmetric activation (i.e., less LH and more RH activation compared to LR reading), thus making judgments to be slower. 


\section{Mental Health \& Human Resilience International Journal}

In the exceptional case of fearful faces in which the TB reading condition enhanced faster detection, we believe that this could be due to the evolutionary importance that exists for detecting (and reacting to) harmful events, in this case by detecting fear on the faces of others. Thus, the recognition of fear, which would be the function of the $\mathrm{LH}$ would be more automatic and require less valencespecific processing. Despite this advantage in speed (lower response time), however, we can see a disadvantage in accuracy (lower performance) as compared to the LR reading condition, presumably because of the lower LH activation.

Considering the above explanation, the results of this study may fit with a supposed symmetric vs. left-sided asymmetric activation generated by reading in different directions.

Concerning gender differences, there were only two, which were significant despite existing evidence for different emotion processing between the sexes [31-33].

Women had a tendency to be faster at recognizing fearful faces, which is in line with results from Han, Gao, Humphreys, and Ge (2008) and Hoffman et al. (2010) $[31,34]$ as well as the theoretical assumptions of Campbell, Muncer, and Bibel (2001) and Fetchenhauer and Buunk (2005) $[35,36]$, and women were better at recognizing happy faces when reading in a TB direction while men were better in the same task when reading in a LR condition.

\section{Conclusion}

The goals of our experiment were to test whether or not reading could have the same effect as a unilateral physical stimulation and to test which emotionprocessing model could better predict its effect. The results could not reaffirm clearly either the $\mathrm{VH}$ or the $\mathrm{RHH}$, but they were in line with predictions made by the working model of Killgore and Yurgelun-Todd (2007) [30], where emotion processing is achieved through two important initial steps. First, the right hemisphere detects an emotionally relevant stimulus, and second, it then sends the stimulus to the left hemisphere where an evaluation process takes place and a valence is attached. In light of this, our experiment supports the idea that reading can function as a unilateral physical stimulation and it also supports the emotion processing model proposed by Killgore and Yurgelun-Todd.

In this study, the Japanese participants were faster and better at recognizing emotional stimuli (except fear) when reading in a left-to-right direction than when they read in a top-to-bottom direction. However, they were worse at correctly identifying neutral faces. There are two ways of interpreting these results from the point of view of different perspectives. From a LR perspective (e.g. from an English reader's perspective), this could mean that in the TB condition, LH activation was inferior and valence attribution to emotional stimuli was hindered. From a TB point of view (e.g. from the perspective of a Japanese reader who only read in a TB direction), however, the inferior performance of LR readers in identifying neutral faces could be viewed as a heightened tendency to see emotions where there are none.

Therefore, based on this pilot study we conclude that it is possible that reading direction can influence the way how we interpret and also construct our subjective understanding of emotions.

\section{Future Research}

As mentioned earlier, further experiments with larger sample sizes and trial numbers are needed to be conducted to validate the findings of this pilot research and, if sufficiently supported, develop an understanding on how direction of reading influences perception of emotions.

In case of its verification in the future we would propose two main ideas for further research. First, we would encourage clinical and health psychologists to consider whether or not reading direction may alter other emotion-related processes such as emotion regulation. Researchers such as Johnstone and Walter (2014) [37] found a laterality effect in emotion regulation after stimulating or disrupting the dorsolateral prefrontal cortex (dlPFC) region of the brain, which is responsible for emotion regulation, through the use of electric currents. Given their results, it is possible that the assumed lateralized activation patterns of the hemispheres aroused during reading could alter emotionregulation capabilities just as electrostimulation/disruption does. According to Ochsner and Gross (2014) [38], the working memory needed for reading seems to share the same dIPFC region with topdown emotion-regulation functions such as attentional deployment, reappraisal, or expression suppression.

Second, given the vast literature on hemispheric lateralization, we believe it would be interesting to test whether reading direction could have any effect on other cognitive systems such as iconic-propositional memory, global-local attention, and divergent-convergent thinking, 


\section{Mental Health \& Human Resilience International Journal}

It is very likely that results from future research will vary and its almost certain that it will take many time and work until we fully understand how the direction of reading affects us. Nevertheless, it is undeniable according to this pilot study that in some way reading direction influences emotion recognition and we believe it's worth researching.

\section{Acknowledgements}

I would like to give my most honest thanks to Junko Momoi and to my brother who both helped me a lot in making this experiment become a reality. Without them it would have been impossible for me to do this research in Japan. I would also like to thank my family and my colleague Dr. Beatrix Labadi for their continuous support, advice and patience during the writing phase of this article. Finally, I would like to thank everyone with whom I got in touch with and provided me with support both professionally and emotionally in this past few years while I was working on this paper.

\section{Statement}

I hereby ensure that the work described has been carried out in accordance with The Code of Ethics of the World Medical Association (Declaration of Helsinki).

\section{References}

1. Schiff BB, Lamon M (1989) Inducing emotion by unilateral contraction of facial muscles: A new look at hemispheric specialization and the experience of emotion. Neuropsychologia 27(7): 923-935.

2. Schiff BB, Lamon M (1994) Inducing emotion by unilateral contraction of hand muscles. Cortex 30(2): 247-254.

3. Ellis AW, Brysbaert M (2010) Split fovea theory and the role of the two cerebral hemispheres in reading: $A$ review of the evidence. Neuropsychologia 48(2): 353365.

4. Jordan TR, Paterson KB (2009) Re-evaluating splitfovea processing in visual word recognition: A critical assessment of recent research. Neuropsychologia 47(12): 2341-2353.

5. Van der Haegen L (2012) Visual word recognition in the fovea (Unpublished doctoral dissertation). Ghent University, Belgium.
6. Pollatsek A, Bolozky S, Well AD, Rayner K (1981) Asymmetries in the perceptual span for Israeli readers. Brain and Language 14(1): 174-180.

7. Brysbaert M (1994) Interhemispheric transfer and the processing of foveally presented stimuli. Behavioural Brain Research 64(1-2): 151-161.

8. O'Regan JK, Jacobs AM (1992) Optimal viewing position effect in word recognition: A challenge to current theory. Journal of Experimental Psychology: Human Perception and Performance 18(1): 185-197.

9. Van der Haegen L, Drieghe D, Brysbaert M (2010) The split fovea theory and the Leicester critique: What do the data say?. Neuropsychologia 48(1): 96-106.

10. Nazir TA, Ben-Boutayab N, Decoppet N, Deutsch A, Frost R (2004) Reading habits, perceptual learning, and recognition of printed words. Brain and Language 88(3): 294-311.

11. Wong YK, Hsiao JH (2012) Reading direction is sufficient to account for the optimal viewing position in reading: The case of music reading. In: $\mathrm{N}$ Miyake, et al. (Eds.), Processing of the 34th Annual Conference of the Cognitive Science Society, Austin, TX: Cognitive Science Society, Pp: 2540-2545.

12. Baijal S, Srinivasan N (2011) Emotional and hemispheric asymmetries in shifts of attention: An ERP study. Cognition and Emotion 25(2): 280-294.

13. Davidson RJ, Mednick D, Moss E, Saron C, Schaffer CE (1987) Ratings of emotion in faces are influenced by the visual field to which stimuli are presented. Brain Cognition 6(4): 403-411.

14. Jansari A, Rodway P, Goncalves S (2011) Identifying facial emotions: Valence specific effects and an exploration of the effects of viewer gender. Brain and Cognition 76(3): 415-423.

15. Gainotti G (1972) Emotional behavior and hemispheric side of the lesion. Cortex 8(1): 41-55.

16. Levy J, Heller W, Banich MT, Burton LA (1983) Asymmetry of perception in free viewing of chimeric faces. Brain Cognition 2(4): 404-419.

17. Torro-Alves N, Sousa JPMD, Fukusima SS (2011) Hemispheric asymmetries on the perception of facial expressions: A divided visual field study. Psicologia USP 22(1): 181-196. 


\section{Mental Health \& Human Resilience International Journal}

18. Schiff BB, Rump SA (1995) Asymmetrical hemispheric activation and emotion: The effects of unilateral forced nostril breathing. Brain and Cognition 29(3): 217-231.

19. Bassel C, Schiff BB (2001) Unilateral vibrotactile stimulation induces emotional biases in cognition and performance. Neuropsychologia 39(3): 282-287.

20. Kajii N, Osaka N (2000) Optimal viewing position in vertically and horizontally presented Japanese words. Perception \& Psychophysics 62(8): 1634-1644.

21. Demaree HA, Everhart DE, Youngstrom EA, Harrison DW (2005) Brain lateralization of emotional processing: Historical roots and a future incorporating "dominance." Behavioral and Cognitive Neuroscience Reviews 4(1): 3-20.

22. Fusar-Poli P, Placentino A, Carletti F, Allen $P$, Landi $P$, et al. (2009) Laterality effect on emotional faces processing: ALE meta-analysis of evidence. Neuroscience Letters 452(3): 262-267.

23. Harmon-Jones E, Allen JJB (1998) Anger and frontal brain activity: EEG asymmetry consistent with approach motivation despite negative affective valence. Journal of Personality and Social Psychology 74(5): 1310-1316.

24. Harmon-Jones E, Sigelman JD, Bohlig A, HarmonJones C (2003) Anger, coping, and frontal cortical activity: The effect of coping potential on angerinduced left frontal activity. Cogn Emot 17(1): 1-24.

25. Harmon-Jones E, Vaughn-Scott K, Mohr S, Sigelman J, Harmon-Jones C (2004) The effect of manipulated sympathy and anger on left and right frontal cortical activity. Emotion 4(1): 95-101.

26. Lyons M J, Akamatsu S, Kamachi MG, Gyoba J (1998) Coding facial expressions with Gabor wavelets. Proceedings of the Third International Conference on Automatic Face and Gesture Recognition: 200-205.

27. Adams RB Jr, Rule NO, Franklin RG Jr, Wang E, Stevenson MT, et al. (2010) Cross-cultural reading the mind in the eyes: An fMRI investigation. J Cogn Neurosci 22(1): 97-108.

28. Baron-Cohen S, Wheelwright S, Hill J, Raste Y, Plumb I (2001) The "Reading the Mind in the Eyes" test revised version: A study with normal adults, and adults with Asperger syndrome or high-functioning autism. Journal of Child Psychology and Psychiatry 42(2): 241-251.

29. Stevenson MT, Soto JA, Adams RB Jr (2012) More than meets the eye: The role of self-identity in decoding complex emotional states. Emotion 12(5): 882-886.

30. Killgore WD, Yurgelun-Todd DA (2007) The righthemisphere and valence hypotheses: Could they both be right (and sometimes left)?. Social Cognitive \& Affective Neuroscience 2(3): 240-250.

31. Hoffmann H, Kessler H, Eppel T, Rukavina S, Traue HC (2010) Expression intensity, gender and facial emotion recognition: Women recognize only subtle facial emotions better than men. Acta Psychol 135(3): 278-283.

32. Rodway P, Wright L, Hardie SM (2003) The valencespecific laterality effect in free viewing conditions: The influence of sex, handedness, and response bias. Brain and Cognition 53(3): 452-463.

33. Van Strien JW, Van Beek S (2000) Ratings of emotion in laterally presented faces: sex and handedness effects. Brain and Cognition 44(3): 645-652.

34. Han S, Gao X, Humphreys GW, Ge J (2008) Neural processing of threat cues in social environments. Human brain mapping 29(8): 945-957.

35. Campbell A, Muncer SJ, Bibel D (2001) Women and crime: An evolutionary approach. Aggression and Violent Behavior 6(5): 481-497.

36. Fetchenhauer D, Buunk BP (2005) How to explain gender differences in fear of crime: Towards an evolutionary approach. Sexualities Evolution \& Gender 7(2): 95-113.

37. Johnstone $\mathrm{T}$, Walter $\mathrm{H}$ (2014) The neural basis of emotion dysregulation. In: James J Gross (Eds.), Handbook of Emotion Regulation 2nd (edn), The Guilford Press, New York, pp: 58-75.

38. Ochsner KN, Gross JJ (2014) The neural bases of emotion and emotion regulation: A valuation perspective. In: James J Gross (Eds.), Handbook of Emotion Regulation 2nd (edn), The Guilford Press, New York, pp: 23-42.

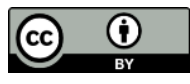

Copyright $($ C Vargha B and Labadi B.
Vargha B and Labadi B. The Effect of Reading Direction on Emotion Recognition: A Pilot Study. Ment Health Hum Resilience Int J 2019, 3(1): 000126. 